\title{
Tissue Factor Expression in the Symptomatic Carotid Plaque
}

\author{
Hani Abdul-Jabar ${ }^{\mathrm{a}, \mathrm{b}}$, Abbas Rashid ${ }^{\mathrm{a}}$, Amir Sadri ${ }^{\mathrm{a}}$, Trevor Paes ${ }^{\mathrm{a}}$
}

\begin{abstract}
Background: The aims of this study were to identify that the differences in the natural history of patients with symptomatic and asymptomatic carotid stenosis may be reflected in differences in the expression of procoagulant protein factors.
\end{abstract}

Methods: Carotid artery plaques were obtained from 33 symptomatic and 4 asymptomatic patients with internal carotid artery stenosis of greater than $70 \%$. These plaques were stained with monoclonal antibody against human tissue factor. Areas of staining for the cap and core were analysed using the analySIS computer programme.

Results: There were 37 patients, of whom 27 were male with a mean age 69.3 years and a range of 53 to 83 years. Statistical analysis using non-parametric tests revealed a significant increase in the area of positive staining for tissue factor in plaques taken from symptomatic patients when compared to those who were asymptomatic $(P=0001)$. Within the symptomatic patients group there was significantly increased tissue factor in the plaque core of those who were the most recently symptomatic $(\mathrm{P}=0.003)$.

Conclusions: The unstable carotid artery plaque is associated with significantly increased tissue factor expression in the cap and core. Plaques from the most recently symptomatic patients have significantly more tissue factor in the core and this may represent part of the mechanism responsible for plaque destabilisation. More research is needed in this important area.

Keywords: Tissue Factor; Carotid stenosis; Stroke; Plaque stability

Manuscript accepted for publication July 9, 2009

${ }^{a}$ Department of Vascular Surgery, The Hillingdon Hospital, Pield Heath Road, Uxbridge, Middlesex UB8 3NN, UK

bCorresponding author: hba999@hotmail.com

doi:10.4021/jocmr2009.07.1250

\section{Introduction}

Stroke is the third commonest cause of death in the UK after coronary disease and cancer, and the principal cause of neurological disability [1]. The annual UK incidence of stroke is 2:1000 and each year 125000 patients will suffer their first stroke [2]. Half of strokes affect patients over 75 years and only $25 \%$ occur in patients under the age of 65 [1]. Stroke patients in the UK utilise $10 \%$ of hospital bed-days and $5 \%$ of annual health expenditure [3]. Stroke mortality within the UK decreased by up to $20 \%$ over the last 30 years [4]. However, because of an increase in the ageing population, the overall incidence of stroke could increase by up to $30 \%$ by 2033 [5]. The vast majority of strokes are ischaemic, of these $80 \%$ affect the carotid territory.

The commonest cause of ischaemic stroke is thromboembolism of the internal carotid artery (ICA). Stenoses develop at the origin of the ICA because this is a complex region with haemodynamic phenomena compromising low shear stress, flow stasis and flow separation that predispose to atherosclerotic plaque formation. Should the plaque undergo acute change (rupture, ulceration, or intraplaque haemorrhage), the inner core of thrombogenic subendothelial collagen is exposed and this predisposes towards the formation of thrombus and the onset of symptoms [6].

\section{Carotid artery disease}

The carotid artery plaque is a dynamic structure. It may be stable and unlikely to produce symptomatic embolization and carotid occlusion or conversely, while not necessarily being any more stenotic, unstable and at high risk of producing symptomatic embolization or carotid occlusion [7].

\section{Mechanism of stroke in symptomatic carotid artery disease}

The majority of strokes are due to embolization from an atherosclerotic plaque or acute occlusion of the carotid artery and propagation of thrombus distally [7], however a small number of strokes can be attributed to hypoperfusion [8]. Low flow alone is not usually sufficient to cause ischaemic 
stroke distal to symptomatic carotid stenosis. Post-stenotic narrowing may be protective because low blood flow distal to the stenosis is insufficient to carry emboli to the brain [9].

\section{Relationship between presenting symptoms and stroke}

Risk factors for an ischaemic stroke include: sex, increasing age, smoking, hypertension, ischaemic heart disease, cardio-embolic source, peripheral vascular disease and diabetes [6]. Presenting symptoms significantly predict outcome after carotid endarterectomy (CEA). Patients with amaurosis fugax (AF) have a significantly better survival than those with transient ischaemic attacks (TIA's), transient strokes or progressive strokes [10]. A history of crescendo TIA's and being female are associated with an increased incidence of perioperative death and stroke within 30 days of the operation. Deaths between 1 and 36 months are associated with both ischaemic heart disease and diabetes in both sexes [10].

\section{Carotid atherosclerotic plaque morphology}

Angiographic plaque surface morphology is used along with the degree of carotid stenosis to identify patients most likely to benefit from CEA and other preventive treatment [11]. MRI angiography, CT scans and colour coded duplex ultrasonography are now very reliable diagnostic techniques and have largely superseded routine arterial angiography for surgical planning [12]. Biasi et al recently showed a relationship between plaque echogenicity and stroke risk. His study demonstrated that the grey scale median of a plaque, measured using a computerised method is associated with the presence or absence of CT-brain infarction. Plaques with high grey scale median (echogenic) were associated with an $11 \%$ incidence of CT-brain infarction, whereby plaques with low grey scale median (echolucent) were associated with a $55 \%$ incidence of CT-brain infarction [13]. Previous studies have already suggested that the presence of CT-brain infarcts is a risk factor for subsequent stroke and death [14-17].

\section{Cellular biology and plaque rupture}

Previous histological examinations have demonstrated subtle differences in the characteristics of atherosclerotic plaque removed from symptomatic patients. In symptomatic patients inflammation is more common, with greater number of macrophages and $\mathrm{T}$ cells detected in the cap of the symptomatic plaque [18-20]. The necrotic core is also placed nearer to the fibrous cap and the minimum cap thickness is less [7].

Smooth muscle cells lay down collagen, the principal connective tissue component of the fibrous cap. Collagen breakdown is dependant on the balance between proteolytic enzymes; matrix metallaproteinases (MMP's) and their in- hibitors; tissue inhibitors of metalloproteinases (TIMP's). High levels of MMP's have been demonstrated at the site of the inflammatory infiltrate in the fibrous cap [21], smooth muscle cell apoptosis has also been demonstrated in unstable carotid plaques [22].

T-cells secrete CD-40 and interleukin-1 (IL-1), which activate macrophages which in turn stimulate the apoptotic activity of MMP's resulting in collagen degradation [7].

\section{Plaque thrombogenicity}

On plaque rupture, exposure of the necrotic core to circulation promotes thrombosis. This appears to be an important mechanism of plaque progression, in addition to embolization. Increased expression of tissue factor, the most important stimulant of the extrinsic clotting cascade, has been demonstrated in plaques from patients with unstable angina or myocardial infarction [23]. In an animal model, plaque rupture is associated with increased tissue factor production from circulating monocytes, which is reduced by treatment with a nitrous oxide precursor [24].

\section{Tissue factor (TF)}

TF is a $47-\mathrm{kD}$ membrane bound, low weight molecular glyco-protein essential for the activation of the extrinsic coagulation pathway. TF receptor is a member of the class 2 cytokine receptor super-family. Growth factors and cytokines present in vascular smooth muscle cells and macrophages regulate the TF expression [25].

TF complexes with factors VII and VIIa, permitting enzymatic activation of factors $\mathrm{X}$ and IX, the substrates for factor VIIa [26], and ultimately leading to the generation of thrombin [26]. TF is strongly induced in activated inflammatory macrophages and T cells. By its ability to bind factor VIIa; TF directly activates the coagulation cascade. Therefore, $\mathrm{TF}$ is a candidate molecule linking plaque inflammation with arterial thromboembolism. In the present study, we carried out an immunocytochemical analysis of TF expression in endarterectomy specimens from 37 patients undergoing CEA for high-grade ICA stenosis and addressed the relationship of TF expression to clinical features of plaque instability.

\section{Subjects and Methods}

\section{Patients}

The study included 37 surgical in-patients enlisted to undergo CEA for high-grade ICA stenosis $(\geq 70 \%$ luminal narrowing). The study was approved by the local ethics review committee and performed in accordance with institutional guidelines. Informed consent was obtained from all 
Table 1. Clinical features of symptomatic and asymptomatic patients with high-grade ICA stenosis ( $\geq 70 \%)$

\begin{tabular}{cll}
\hline & Age (mean \pm SD) & Sex (Male) \\
\hline All Patients $(\mathrm{n}=37)$ & $69 \pm 7$ & 27 \\
Asymptomatic $(\mathrm{n}=4)$ & $72 \pm 4$ & 1 \\
Symptomatic $(\mathrm{n}=33)$ & $69 \pm 9$ & 26 \\
Time Since Symptoms & Number & Sex (Male) \\
Asymptomatic & 4 & 1 \\
1-6 Months & 17 & 16 \\
$<1$ Month & 16 & 10 \\
Type of Symptoms & Number & Sex (Male) \\
Asymptomatic & 4 & 1 \\
TIA/AF & 26 & 5 \\
Stroke & 7 & 21 \\
\hline
\end{tabular}

patients. Baseline characteristics of the study population are provided in Table 1. The degree of stenosis was determined by Standardised Imaging and Doppler Criteria for Cerebrovascualr Diagnosis using Duplex Sonography [27]. During surgery intra-venous heparin was given to all patients as an anti-coagulant.

\section{Histological procedure and immunocytochemistry}

After longitudinal arteriotomy, the carotid atherosclerotic plaque was excised en bloc (routine endarterectomy), fixed immediately in buffered formalin, decalcified, and transversely sectioned at 5-mm intervals [28]. Each 5-mm tissue bloc was embedded separately into paraffin. For immunocytochemistry, 3-mm sections were mounted onto gelatine-coated slides. After deparaffinization, sections were incubated with monoclonal antibody $(\mathrm{mAb})$ against human tissue factor (No.4508, American Diagnostica Inc) at 1:200 dilution, followed by goat anti-mouse (Vector Laboratories) and the ABC ELITE kit reagents (Vector) with diaminobenzidine as a substrate. For antigen retrieval all sections were microwaved in $10 \mathrm{mmol} / \mathrm{L}$ sodium citrate buffer, $\mathrm{pH} 6.0$, for 10 minutes before staining.

Absence of tissue factor antibody was regarded as a negative control, while a positive control was the presence of tissue factor antibody in a human colon cancer tissue. Mayer's hematoxilyn was used to counter-stain all the sections to enable the histopathologist to review the slides.

\section{Quantification}

The analySIS ${ }^{\mathrm{TM}}$ computer program was used to calculate the total area of positive TF staining within the cap and core of each specimen.

\section{Statistical Analysis}

Paired sample T-tests were not used to analyse the data, as it was not normally distributed (v values of the ShapiroFrancia $\mathrm{W}$ normality test were outside the range of 2.0-2.8, $\mathrm{v}$ value of cap $=3.6$, v value of core $=1.8$ ). The non-parametric Mann-Whitney $U$ test was used to investigate the relationship between the extent of TF expression (median $\mathrm{TF}+$ section area in the cap and core of each plaque) and the occurrence of ischaemic symptoms. Kruskal-Wallis nonparametric test was used to study the relationship between $\mathrm{TF}$ expression (median $\mathrm{TF}+$ section area in the cap and core of each plaque) and the time since symptoms. P values of $<0.05$ were considered indicative of statistically significant findings. Measurement errors were calculated to account for the natural variation of the measurement process using the repeatability method [29]; values calculated were not significant (mean difference $=915.4$, mean within-subject standard deviation $=647.3)$.

\section{Results}

TF expression was found in all plaques, however there was a significant difference in the area of positive staining in plaques from symptomatic (Fig. 1) and asymptomatic (Fig.

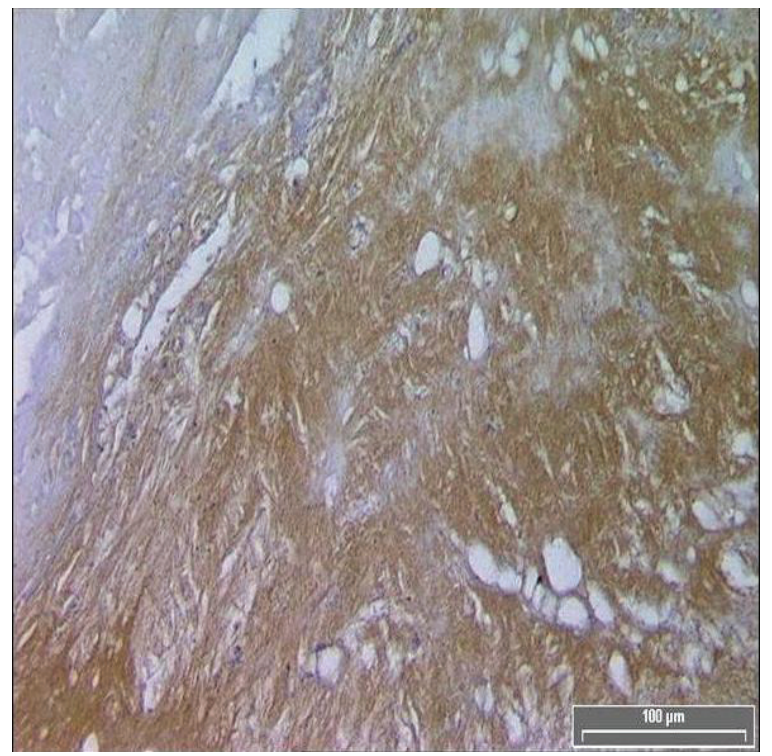

Figure 1. Diffuse TF immuno-reactivity in the core of a symptomatic patient. 


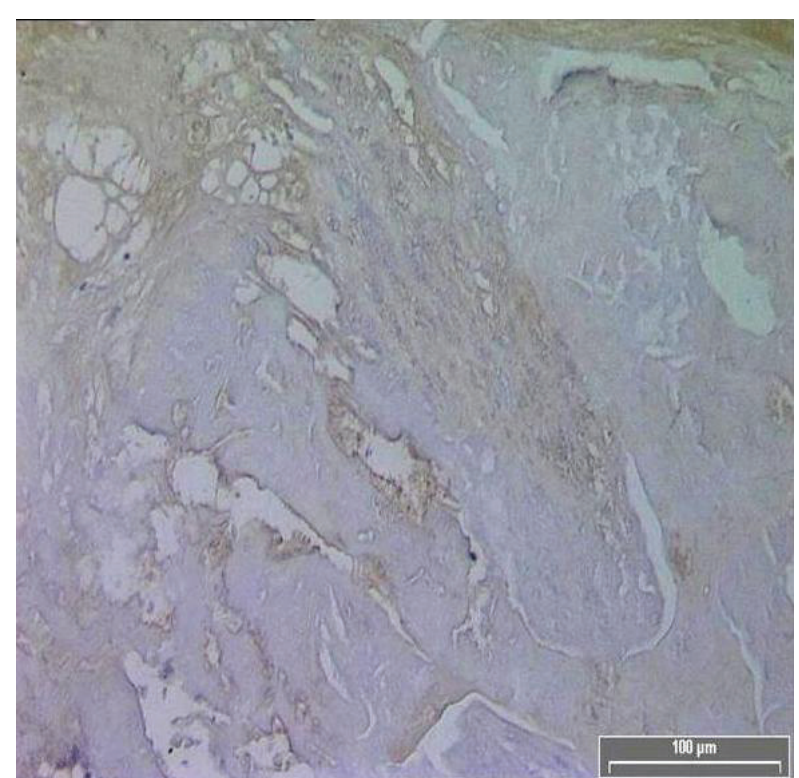

Figure 2. Diminished TF immuno-reactivity in the core of an asymptomatic patient.

2) patients. The resulting $P$ values (Table 2) correspond to Mann-Whitney U non-parametric test with units of $\mathrm{mm} 2 \mathrm{ex}$ pressing the median and the interquartile range. (Fig. 3, 4)

The patients were then divided into groups according to the length of time between the last acute event and the CEA. Using the Kruskal-Wallis non-parametric test (Table 3 ); the expression of TF in the core was shown to be significantly increased in plaques from the most recently symptomatic patients. (Fig. 5).

\section{Discussion}

In this study we have shown that in the most recently symptomatic carotid artery plaque there is an increased expression of TF when compared to the asymptomatic carotid artery plaque. Most TF immuno-reactivity was localised diffusely in the acellular necrotic core and in regions within the atheroma's fibrous cap. Our data therefore strongly suggest that $\mathrm{TF}$ induction at various regions of the plaque may play an important role in the destabilisation of high-grade ICA stenosis.

The mechanism responsible for thrombus formation associated with ruptured atherosclerotic plaques is not well understood. One widely held view is that rupture or fissuring of an atherosclerotic plaque results in exposure of collagen and other extracellular proteins to blood-borne platelets, which adhere to these elements via specific receptors and become activated [30]. Activated platelets release ADP and thrombin, bind fibrinogen, and activate other platelets, thereby producing local thrombosis in and on the arterial wall [25].
Exposure of TF activity to coagulation factors may either occur directly at the intimal surface or may result from plaque rupture leading to the release of macrophage-bound or extracellular material from deeper parts of the plaque to the blood stream [31]. The rupture of complicated plaques has been suggested to be due to the expression of MMP's that degrade extracellular matrix components and thereby weaken the fibrous cap [21, 32]. A recent study by Loftus et al [33] showed a correlation between MMP-9 expression and carotid plaque destabilisation. Thus, it is an intriguing hypothesis that the concerted action of MMP-9 and TF may be a key mechanism of plaque destabilisation in cerebrovascular disease patients who are at high risk of stroke.

TF pathway inhibitor (TFPI) is found in vascular endothelium and smooth muscle cells as well as in platelets, blood monocytes, and macrophages $[34,35]$. It provides physiological inhibition of TF-initiated coagulation by binding to factors Xa and the TF-factor VIIa complex in a 2-step process. In atherosclerotic carotid arteries, TF expression is abundant, whereas TFPI expression is limited in up to $30 \%$ of plaques, resulting in predominant TF activity [36].

Evidence also suggests that TF may have a variety of other actions in addition to initiating thrombin formation. For example, TF: VIIa induces the activation of mitogenactivated protein kinase (MAP-Kinase) in a baby hamster kidney [37] and evokes intracellular $\mathrm{Ca}+2$ mobilisation in human endothelial cells [38], which may lead to cell proliferation.

Human atherosclerotic plaque tissue from CEA specimens express TF messenger-RNA and protein in a few intimal smooth muscle cells, as well as in a few macrophagelike cells adjacent to cholesterol clefts [25]. Wilcox et al [39] showed that TF antigen seemed to occur extracellularly. Annex et al [40] demonstrated antigenic TF in coronary atherectomy specimens but, as with previous immunohistochemical studies, TF was seen in only approximately one third of specimens. When using digoxigenin labelled factors VIIa and X, Thiruvikraman et al [25] showed diffuse TF activity both intracellularly (endothelial cells, smooth muscle cells,

Table 2. TF positive staining area in asymptomatic and symptomatic Patients

\begin{tabular}{llll}
\hline & Asymptomatic & Symptomatic & $\begin{array}{l}\text { P } \\
\text { Value }\end{array}$ \\
\hline \multirow{2}{*}{ TF Cap } & $\begin{array}{l}379.1 \\
(301.4-703.2)\end{array}$ & $\begin{array}{l}37296.2 \\
(32067.6-43648.7)\end{array}$ & 0.001 \\
& & & \\
TF Core & 399.8 & 52689.2 & 0.001 \\
& $(282.2-710.4)$ & $(39798.5-70827.0)$ & \\
\hline
\end{tabular}

*All units are $\mathrm{mm}^{2}$ and expressed as a median (interquartile range). 
TF area positive staining $\mu \mathrm{m}^{2}$

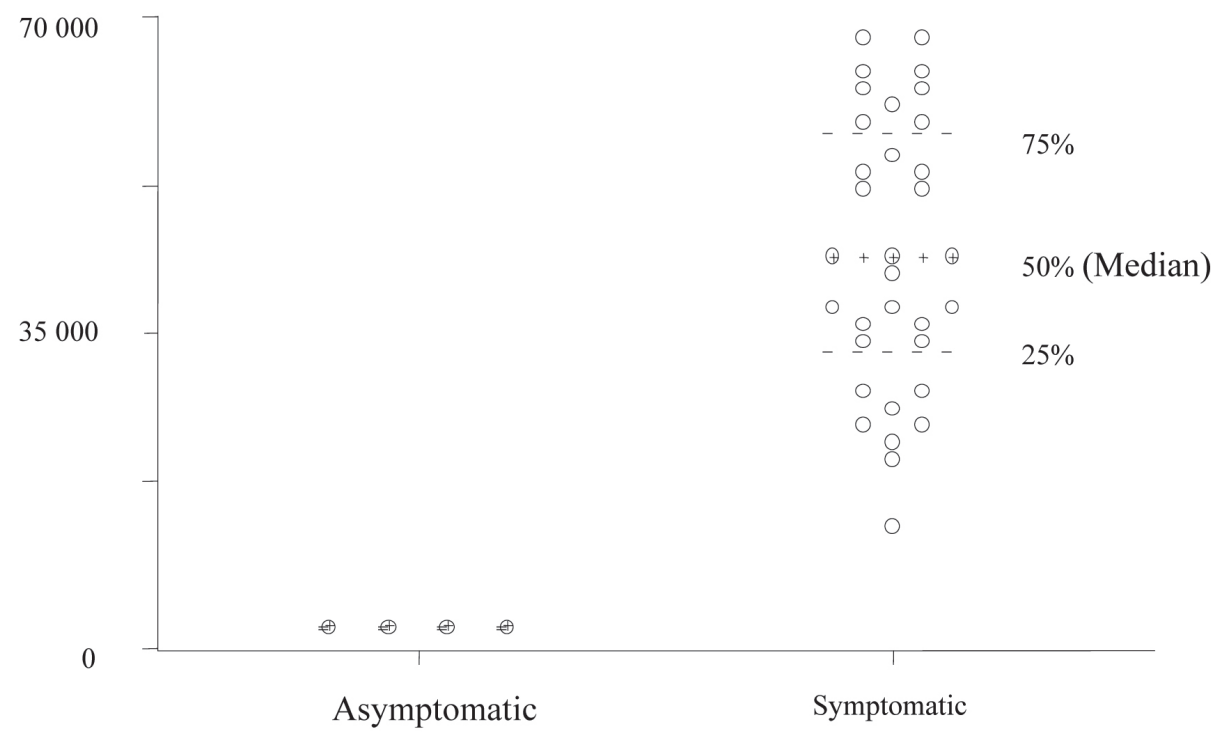

Figure 3. Scatter Plot of TF positive staining area in the Plaque Cores of Asymptomatic and Symptomatic Patients (units are expressed in $\mathrm{mm}^{2}$ ).

and macrophages) and extracellularly (lipid-rich core and fibrous matrix) in virtually all human atherosclerotic plaques taken from various sites.

A potential limitation of our study arises from the fact that the sensitive immuno-histochemical staining procedure used for the detection of TF antigen does not allow direct conclusions with respect to the actual presence of TF bio- activity. However in a comparative study, Jander et al [31] used a similar TF-specific antibody for the in-situ detection of TF and observed essentially identical staining patterns with a similar type of detection reagent; TF specific antibody bound to the atheroma's acellular lipid core. It is therefore likely that the TF immuno-reactivity detected in our study indeed reflects TF binding activity for its physiologically rel-

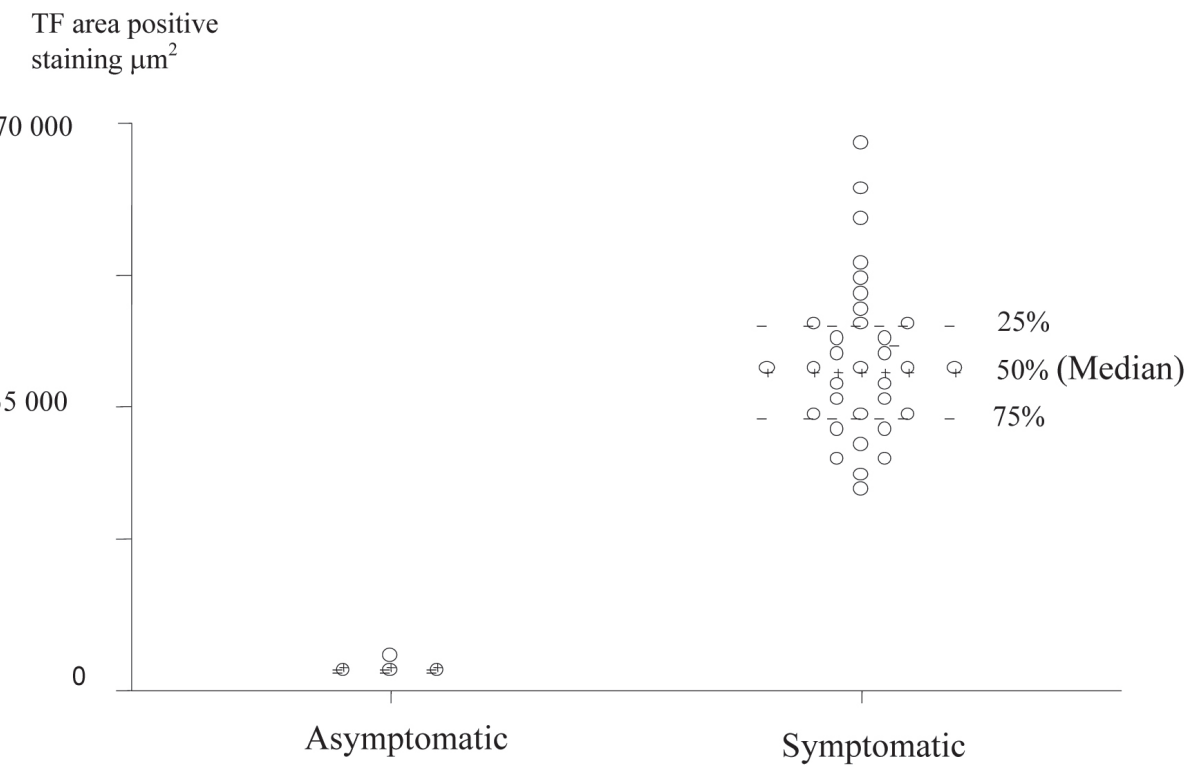

Figure 4. Scatter Plot of TF positive staining area in the Plaque Caps of Asymptomatic and Symptomatic Patients (units are expressed in $\mu \mathrm{m}^{2}$ ). 
Table 3. Increased TF positive staining Area in the plaque cores of the most recently symptomatic patients

\begin{tabular}{lllll}
\hline & Asymptomatic & $<$ Month & 1-6 Months & P Value \\
\hline \multirow{3}{*}{ TF Core } & 399.8 & 64386.2 & 46286.7 & 0.003 \\
& $(282.2-710.4)$ & $(38320.6-75970.5)$ & $(39698.5-63462.7)$ & \\
\hline
\end{tabular}

evant ligands. However, additional studies using TF bioassays will be necessary to definitively clarify the role of TF in ICA plaque destabilisation.

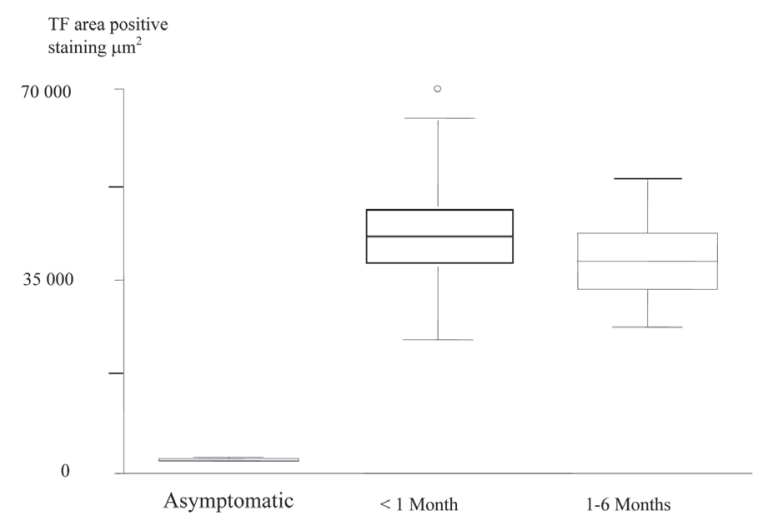

Figure 5. Box and Whisker plots, which show, increased TF Expression in the Plaque Cores of the most recently Symptomatic Patients.

\section{References}

1. Warlow CP. Disorders of the cerebral circulation. In: Walton J (ed) Brain's diseases of the nervous system. Oxford: Oxford University Press, 1993, pp. 197-210.

2. Bamford J, Sandercock P, Dennid M, Burn J, Warlow CP. A prospective study of acute cerbrovascular disease in the community. The Oxfordshire Community Stroke Project 1981-1986. (I) Methodology, demography and incident cases of first ever stroke. J Neurol Neurosurg Psych 1988; 51:1373-1380.

3. Dunbabin D, Sandercock P. Stroke prevention. Hospital Update 1992; July: 540-545.

4. Modan B, Wagener DK. Some epidemiological aspects of stroke: mortality/morbidity trends, age, sex, race, socioeconomic status. Stroke 1992;23(9):1230-1236.

5. Malmgren R, Bamford J, Warlow C, Sandercock P, Slattery J. Projecting the number of patients with first ever strokes and patients newly handicapped by stroke in England and Wales. BMJ 1989;298(6674):656-660.

6. Beard J, Gaines P. Extracranial Cerebrovascular Dis- ease. In: W.B Saunders Vascular and Endovascular Surgery, 2nd edition 2000, pp. 327-365.

7. Golledge J, Greenhalgh RM, Davies AH. The symptomatic carotid plaque. Stroke 2000;31(3):774-781.

8. Ringelstein EB, Sievers C, Ecker S, Schneider PA, Otis SM. Noninvasive assessment of CO2-induced cerebral vasomotor response in normal individuals and patients with internal carotid artery occlusions. Stroke 1988;19(8):963-969.

9. Rothwell PM, Warlow CP. Low risk of ischemic stroke in patients with reduced internal carotid artery lumen diameter distal to severe symptomatic carotid stenosis: cerebral protection due to low poststenotic flow? On behalf of the European Carotid Surgery Trialists' Collaborative Group. Stroke 2000;31(3):622-630.

10. Golledge J, Cuming R, Beattie DK, Davies AH, Greenhalgh RM. Influence of patient-related variables on the outcome of carotid endarterectomy. J Vasc Surg 1996;24(1):120-126.

11. Rothwell PM, Warlow CP. Prediction of benefit from carotid endarterectomy in individual patients: a risk-modelling study. European Carotid Surgery Trialists' Collaborative Group. Lancet 1999;353(9170):2105-2110.

12. Bohmeke T, Lange R, Mumme A, Barbera L. Carotid stenosis- a non-invasive diagnostic approach should receive preference. Fortschritte der Medizin 1998; 116:2024.

13. Biasi GM, Sampaolo A, Mingazzini P, De Amicis P, El-Barghouty N, Nicolaides AN. Computer analysis of ultrasonic plaque echolucency in identifying high risk carotid bifurcation lesions. Eur J Vasc Endovasc Surg 1999; 17(6):476-479.

14. Predictors of major vascular events in patients with a transient ischemic attack or nondisabling stroke. The Dutch TIA Trial Study Group. Stroke 1993;24(4):527531.

15. Evans GW, Howard G, Murros KE, Rose LA, Toole JF. Cerebral infarction verified by cranial computed tomography and prognosis for survival following transient ischemic attack. Stroke 1991;22(4):431-436.

16. Nicoladies AN, Kalodiki E, Ramaswani G et al. The significance of cerebral infarcts on $\mathrm{CT}$ scans in patients with transient ischaemic attack. In: Bernstein EF, Callow AD, Nicoladies AN, Shifrin EG. Cerebral Revascularization. London Med-Orion, 1993:159-163. 
17. van Latum JC, Koudstaal PJ, Venables GS, van Gijn J, Kappelle LJ, Algra A. Predictors of major vascular events in patients with a transient ischemic attack or minor ischemic stroke and with nonrheumatic atrial fibrillation. European Atrial Fibrillation Trial (EAFT) Study Group. Stroke 1995;26(5):801-806.

18. Carr S, Farb A, Pearce WH, Virmani R, Yao JS. Atherosclerotic plaque rupture in symptomatic carotid artery stenosis. J Vasc Surg 1996;23(5):755-765; discussion 765-756.

19. Bassiouny HS, Sakaguchi Y, Mikucki SA, McKinsey JF, Piano G, Gewertz BL, Glagov S. Juxtalumenal location of plaque necrosis and neoformation in symptomatic carotid stenosis. J Vasc Surg 1997;26(4):585-594.

20. Jander S, Sitzer M, Schumann R, Schroeter M, Siebler M, Steinmetz H, Stoll G. Inflammation in high-grade carotid stenosis: a possible role for macrophages and $\mathrm{T}$ cells in plaque destabilization. Stroke 1998;29(8):16251630 .

21. Galis ZS, Sukhova GK, Lark MW, Libby P. Increased expression of matrix metalloproteinases and matrix degrading activity in vulnerable regions of human atherosclerotic plaques. J Clin Invest 1994;94(6):2493-2503.

22. Best PJ, Hasdai D, Sangiorgi G, Schwartz RS, Holmes DR, Jr., Simari RD, Lerman A. Apoptosis. Basic concepts and implications in coronary artery disease. Arterioscler Thromb Vasc Biol 1999;19(1):14-22.

23. Ardissino D, Merlini PA, Ariens R, Coppola R, Bramucci E, Mannucci PM. Tissue-factor antigen and activity in human coronary atherosclerotic plaques. Lancet 1997;349(9054):769-771.

24. Corseaux D, Le Tourneau T, Six I, Ezekowitz MD, Mc Fadden EP, Meurice T, Asseman P, et al. Enhanced monocyte tissue factor response after experimental balloon angioplasty in hypercholesterolemic rabbit: inhibition with dietary L-arginine. Circulation 1998;98(17):17761782.

25. Thiruvikraman SV, Guha A, Roboz J, Taubman MB, Nemerson Y, Fallon JT. In situ localization of tissue factor in human atherosclerotic plaques by binding of digoxigenin-labeled factors VIIa and X. Lab Invest 1996;75(4):451-461.

26. Rapaport SI, Rao LV. Initiation and regulation of tissue factor-dependent blood coagulation. Arterioscler Thromb 1992;12(10):1111-1121.

27. Baker JD et al. Standardised Imaging and Doppler for Cerebrovascular Diagnosis using Duplex Sonography. Presented at the 1986 AIUM, Las Vegas, Nevada USA.

28. Sitzer M, Muller W, Siebler M, Hort W, Kniemeyer HW, Jancke L, Steinmetz H. Plaque ulceration and lumen thrombus are the main sources of cerebral microemboli in high-grade internal carotid artery stenosis. Stroke 1995;26(7):1231-1233.

29. Bland JM, Altman DG. Measurement error. BMJ
1996;312(7047):1654.

30. van Zanten GH, de Graaf S, Slootweg PJ, Heijnen HF, Connolly TM, de Groot PG, Sixma JJ. Increased platelet deposition on atherosclerotic coronary arteries. J Clin Invest 1994;93(2):615-632.

31. Jander S, Sitzer M, Wendt A, Schroeter M, Buchkremer M, Siebler M, Muller W, et al. Expression of tissue factor in high-grade carotid artery stenosis: association with plaque destabilization. Stroke 2001;32(4):850-854.

32. Halpert I, Sires UI, Roby JD, Potter-Perigo S, Wight TN, Shapiro SD, Welgus HG, et al. Matrilysin is expressed by lipid-laden macrophages at sites of potential rupture in atherosclerotic lesions and localizes to areas of versican deposition, a proteoglycan substrate for the enzyme. Proc Natl Acad Sci U S A 1996;93(18):9748-9753.

33. Loftus IM, Naylor AR, Goodall S, Crowther M, Jones L, Bell PR, Thompson MM. Increased matrix metalloproteinase- 9 activity in unstable carotid plaques. A potential role in acute plaque disruption. Stroke 2000;31(1):4047.

34. Bajaj MS, Kuppuswamy MN, Saito H, Spitzer SG, Bajaj SP. Cultured normal human hepatocytes do not synthesize lipoprotein-associated coagulation inhibitor: evidence that endothelium is the principal site of its synthesis. Proc Natl Acad Sci U S A 1990;87(22):8869-8873.

35. Caplice NM, Mueske CS, Kleppe LS, Peterson TE, Broze GJ, Jr., Simari RD. Expression of tissue factor pathway inhibitor in vascular smooth muscle cells and its regulation by growth factors. Circ Res 1998;83(12):12641270.

36. Caplice NM, Mueske CS, Kleppe LS, Simari RD. Presence of tissue factor pathway inhibitor in human atherosclerotic plaques is associated with reduced tissue factor activity. Circulation 1998;98(11):1051-1057.

37. Poulsen LK, Jacobsen N, Sorensen BB, Bergenhem NC, Kelly JD, Foster DC, Thastrup O, et al. Signal transduction via the mitogen-activated protein kinase pathway induced by binding of coagulation factor VIIa to tissue factor. J Biol Chem 1998;273(11):6228-6232.

38. Rottingen JA, Enden T, Camerer E, Iversen JG, Prydz $\mathrm{H}$. Binding of human factor VIIa to tissue factor induces cytosolic $\mathrm{Ca} 2+$ signals in J82 cells, transfected COS-1 cells, Madin-Darby canine kidney cells and in human endothelial cells induced to synthesize tissue factor. J Biol Chem 1995;270(9):4650-4660.

39. Wilcox JN, Smith KM, Schwartz SM, Gordon D. Localization of tissue factor in the normal vessel wall and in the atherosclerotic plaque. Proc Natl Acad Sci U S A 1989;86(8):2839-2843.

40. Annex BH, Denning SM, Channon KM, Sketch MH, Jr., Stack RS, Morrissey JH, Peters KG. Differential expression of tissue factor protein in directional atherectomy specimens from patients with stable and unstable coronary syndromes. Circulation 1995;91(3):619-622. 